\title{
FG Sge as a New-Born Carbon Star
}

\author{
TAKASHI IIJIMA \\ Astronomical Observatory of Padova, Asiago, Italy
}

The spectral type of the post-AGB star FG Sagittae rapidly changed from $\mathrm{B} 4 \mathrm{I}$ in the $1950 \mathrm{~s}$ to $\mathrm{K} 2 \mathrm{Ib}$ in the 1980s. The Swan bands of the $\mathrm{C}_{2}$ molecule have been detected in some spectra taken in 1981 and later. This star seems to have become a carbon star as expected in theories of the evolution of post-AGB stars. The recent spectra, however, are much different from those of normal carbon stars. The absorption lines of $\mathrm{Ba}$ II, Sr II, La II etc. are unusually deep, while those of the iron group are weak. In most spectra the $\mathrm{CN}$ bands are absent even when the $\mathrm{C}_{2}$ bands are easily visible. Since a weak CN band at $4215 \AA$ was detected in a spectrum taken on 23 April 1994, the absence of $\mathrm{CN}$ bands in the other spectra may not have been due to a lack of nitrogen atoms. There might have been a peculiar atmospheric condition in which the $\mathrm{C}_{2}$ molecule was formed, but not the $\mathrm{CN}$ molecule. Large spectral variations have been observed during the photometric decline which started in August 1992.

The full text has appeared in MNRAS 283, 141, 1996. 